\title{
Confidencialidad e historia clínica. Consideraciones ético-legales
}

\section{Confidentiality and the medical record. Ethical-legal considerations}

\author{
J. Antomás ${ }^{1}$, S. Huarte del Barrio ${ }^{2}$
}

\section{RESUMEN}

La confidencialidad es un aspecto clave de la relación entre profesionales sanitarios y pacientes. Supone la cesión del paciente de una parte reservada de sí mismo y los principios éticos de autonomía y no maleficencia están íntimamente ligados con su preservación. También numerosas normas legales obligan al secreto profesional, imponiendo en algunos casos duras sanciones a menudo desconocidas.

Al ser la historia clínica el documento donde la relación con el paciente queda reflejada, requiere de una protección extraordinaria por la naturaleza especialmente sensible de la información en ella contenida. Es preceptivo para el profesional que la elabora conocer algunos aspectos básicos de las leyes fundamentales que la regulan en diferentes aspectos. En este artículo se hace un repaso por cuestiones que a veces resultan conocidas en abstracto, pero que están recogidas en la normativa legal, como son la propiedad de la historia clínica, las anotaciones subjetivas, su custodia y rectificación o quién y para qué se puede acceder a la misma.

Palabras clave. Confidencialidad. Historia Clínica. Bioética.

\begin{abstract}
Confidentiality is a key aspect in the relationship between health professionals and patients. It involves the cession by the patient of a reserved part of herself and the ethical principles of autonomy and non-maleficency are closely linked to its preservation. There are also numerous legal regulations that make professional secrecy obligatory, in some cases imposing hard sanctions that are often not known about.

As the medical record is the document where the relationship with the patient is recorded, it requires extraordinary protection due to the especially sensitive nature of the information it contains. It should be compulsory that the professional who works with it should know some basic aspects of the fundamental laws that regulate it in different aspects. This article reviews questions that at times are only known in the abstract, but that are contained in the legal regulations, such as ownership of the medical record, subjective annotations, its custody and rectification or who can have access to it and why.
\end{abstract}

Key words. Confidentiality. Medical records. Bioethics.
1. Unidad de Hospitalización Psiquiátrica "A”. Complejo Hospitalario de Navarra.

2. Servicio de Emergencias rurales. Irurzun. Navarra.

Recepción: 17 de diciembre de 2010

Aceptación provisional: 21 de enero de 2011

Aceptación definitiva: 28 de enero de 2011

\author{
Correspondencia \\ Javier Antomás Osés \\ Jefe de Unidad de Enfermería \\ Unidad de Hospitalización Psiquiátrica "A" \\ Complejo Hospitalario de Navarra \\ $\mathrm{C} /$ Irunlarrea, 3 \\ 31008 Pamplona (Navarra) \\ E-mail: xabier.antomas.oses@navarra.es
}




\section{INTRODUCCIÓN}

El secreto profesional en el ejercicio de la medicina ha sido un tema de interés a lo largo de la historia. Desde tiempos inmemoriales se ha tenido un especial cuidado en mantener la reserva de la información recabada. Ya en el siglo IV a.C. se formula el "Juramento Hipocrático" que entre sus cinco obligaciones incluye la siguiente:

"Guardaré silencio sobre todo aquello que en mi profesión, o fuera de ella, oiga o vea en la vida de los hombres que no deban ser públicos, manteniendo estas cosas de manera que no se pueda hablar de ellas". (Juramento Hipocrático. Atribuido al médico griego Hipócrates de Cos -Cos, c. 460 a.C.- Tesalia c. 370 a.C.)

Este punto del juramento, presente en la tradición médica occidental a lo largo de los siglos es recogido y adaptado en los códigos deontológicos de las profesiones sanitarias. La Asociación Médica Mundial preconiza en 1948 "Guardar y respetar los secretos confiados a mí, incluso después del fallecimiento del paciente" (2 $2^{\mathrm{a}}$ Asamblea General de la A.M.M. Declaración de Ginebra, Suiza. Septiembre de 1948).

En la incipiente disciplina de enfermería se redacta en 1893 el "Juramento de Florence Nightingale" en honor a la considerada fundadora de la enfermería moderna, que todavía vivía por aquel entonces. En él se lee "Consideraré confidencial toda información personal que me sea revelada y todos los asuntos familiares de los que tenga conocimiento en el ejercicio de mi profesión." [Gretter L.E. et al. The Florence Nightingale Pledge. 1893 Farrand Training School for Nurses, Detroit.] También hace alusión a ello el Consejo Internacional de Enfermeras desde 1953'.

Tanto la Declaración Universal de los Derechos Humanos en su artículo $12^{2}$, como la Constitución Española en el $18^{3}$ tratan la intimidad como un derecho fundamental del individuo.

En 1997, España suscribió el "Convenio para la protección de los derechos humanos y la dignidad del ser humano con respecto a las aplicaciones de la Biología y la Medicina" (Convenio de Oviedo) ${ }^{4}$, que reconoce el derecho de las personas al respeto de su vida privada en asuntos de salud, así como a conocer toda la información obtenida respecto a su salud, salvo las restricciones que establezca la ley.

Las principales normas que reconocen y regulan el derecho a la intimidad en el marco sanitario son la Ley 14/1986 General de Sanidad (art. 10.3) $)^{5}$, la ley Orgánica 15/1999, de 13 de diciembre, de Protección de Datos de Carácter Personal ${ }^{6}$ y su Reglamento de Desarrollo ${ }^{7}$, la Ley 41/2002 de Autonomía del Paciente ${ }^{8}$ y en Navarra la Ley Foral 17/2010 de Derechos y Deberes de las Personas en Materia de Salud ${ }^{9}$.

Cabe destacar que en 2002 estas cuestiones ya se regularon en Navarra en la Ley $11 / 2002$, de 6 de mayo, sobre los derechos del paciente a las voluntades anticipadas, a la información y a la documentación clínica ${ }^{10}$. Esta norma, pionera en el Estado Español, fue modificada parcialmente por la Ley 41/2002 y derogada por la Ley Foral $17 / 2010$.

Por supuesto en la actualidad los códigos deontológicos de las organizaciones colegiales de medicina ${ }^{11}$ y enfermería ${ }^{12}$ recogen la obligación de guardar el secreto profesional.

\section{CONFIDENCIALIDAD}

El diccionario de la Real Academia Española de la lengua define intimidad en su segunda acepción como "Zona espiritual íntima y reservada de una persona o de un grupo, especialmente de una familia" y privacidad como "Ámbito de la vida privada que se tiene derecho a proteger de cualquier intromisión"13. En la vida privada cabe aquello que no se quiere que sea del normal conocimiento y la intimidad forma en ella un núcleo esencial que protegemos con más fuerza ${ }^{14}$.

Respecto a confidencial, se define como "Que se hace o se dice en confianza o con seguridad recíproca entre dos o más personas"13. 
Hablando en términos de bioética, podemos ver principalmente dos principios comprometidos en lo referente a la preservación del secreto: la autonomía y la no maleficencia.

El paciente que acude a un centro sanitario establece una relación quid pro quo en la que deberá ceder información a cambio de una adecuada atención. Atendiendo a su principio de autonomía es libre de revelar la cantidad de datos que estime oportuna, pero esta autonomía se ve limitada por el deber de dar información veraz y suficiente. Así lo recoge diferente legislación sanitaria, como la Ley Foral 17/2010 en su artículo 74.2 "Todas las personas tienen el deber de facilitar los datos sobre su estado físico o sobre su salud que sean considerados necesarios para el proceso asistencial o por razones de interés general, de manera leal y verdadera". La única protección de su intimidad que el paciente tiene ante esta obligación es la discreción del profesional. Se entiende que hay un acuerdo tácito que obliga moralmente al silencio y que también lo recoge la ley. Nos encontramos ante una doble obligación, ética y legal ${ }^{15}$.

Debemos considerar la información privada del paciente un don, en tanto que entrega una parte de sí mismo, en cierto modo única que ha de ser tratada con lealtad sin compartirla con otros ${ }^{14}$. Es solo el propio interesado quien puede decidir qué información pertenece a su más estricta intimidad, según sus propios criterios ${ }^{16}$.

Existe además un deber moral de fidelidad para con el paciente. Se deben respetar los acuerdos a que se llegan (pacta sunt servanda), y esto incluye el acuerdo implícito acerca de la confidencialidad de la información que se maneja en la actuación sanitaria. La relación de los pacientes con los profesionales de la salud se basa necesariamente en la confianza. El usuario debe revelar datos que pertenecen a la esfera de lo íntimo para que quien le atiende cuente con la información necesaria para abordar su proceso. Si esa confianza se quiebra la relación se deteriorará. Esta es la razón básica de carácter práctico que nos impele a mantener el secreto profesional. Debe tenerse en cuenta que la obligación de secreto no solo abarca la información referente al estado de salud del paciente, sino a cualquier dato sobre su vida privada que se conozca durante la atención en salud. Si el profesional no respeta la reserva de lo revelado por el paciente y difunde información privada del mismo, puede provocarle graves daños en diferentes esferas de su vida que serán como mínimo de tipo moral, quebrantando el principio de no maleficencia.

El legislador es sensible al derecho a la confidencialidad de la información y lo recoge en las normas relativas a los derechos de los pacientes en el artículo 7 de la Ley de Autonomía del Paciente. En la Ley Foral $17 / 2010$ lo desarrolla en el 31 y siguientes para proteger explícitamente los siguientes aspectos de la confidencialidad.

- Derecho a limitar la grabación y difusión de registros iconográficos (art. 32). Prohíbe la grabación o fotografía de usuarios de centros o servicios sanitarios sin su expreso consentimiento una vez informados de la finalidad de las imágenes.

- Confidencialidad de los datos genéticos (art. 33). Protege la confidencialidad del patrimonio genético. Debiendo velar las autoridades sanitarias para que nadie pueda ser objeto de discriminación a causa de sus características genéticas.

- Confidencialidad de otros datos especialmente protegidos (art. 34). Los datos referidos a la ideología, religión, creencias, origen racial, vida sexual, al hecho de haber sido objeto de malos tratos y, cuantos datos puedan tener especial relevancia para la salvaguarda de la intimidad personal y familiar deben ser especialmente protegidos. (Sin menoscabo del cumplimiento de los deberes legales de comunicación o denuncia ante cualquiera de los supuestos previstos por la legislación).

Se entiende que la presencia de un intérprete de lengua de signos, familiar o persona de confianza con consentimiento del paciente no vulnera el derecho a la confidencialidad. 
En nuestro medio en ocasiones se desconoce o no se da importancia a la confidencialidad, sin plantearse las repercusiones que puede tener su quebranto ${ }^{17}$. Conversaciones de ascensor ${ }^{18,19}$, comentarios en el centro de trabajo ${ }^{20}$, en el transporte público o descuidos al dejar a la vista documentación con información de pacientes son situaciones que se dan en el medio hospitalario. Informar en lugares inadecuados como pasillos o sin consultar al propio paciente ${ }^{21,22}$ también son prácticas difíciles de erradicar. Costumbres como buscar o pedir a un compañero información sobre conocidos o comentar en casa la situación de amigos de la familia ${ }^{17,23}$ deberían pasar a ser casos excepcionales.

\section{HISTORIA CLÍNICA, ÉTICA Y LEGISLACIÓN}

La definición legal de historia clínica es "el conjunto de documentos que contienen los datos, valoraciones e informaciones de cualquier índole sobre la situación y la evolución clínica de un paciente a lo largo del proceso asistencial"s.

Se trata de un conjunto de documentos que va a ser la base de la atención sanitaria y el reflejo de la relación entre uno o varios profesionales de la salud y el paciente. En ella queda plasmada gran cantidad de información sensible de carácter personal. Su uso adecuado, custodia y cumplimentación son problemas a los que se enfrentan los profesionales sanitarios a diario, que están regulados por diversas normas legales así como por obligaciones de carácter ético que conviene conocer.

Los principios deontológicos implicados en relación con la protección de los datos sanitarios contenidos en la historia clínica son:

a) Sobriedad, en cuanto a consignar sólo las informaciones realmente necesarias para la atención sanitaria.

b) Transparencia en la gestión y acceso a la historia clínica (también para el paciente).

c) Responsabilidad, íntimamente ligada con la no maleficencia por las conse- cuencias para el paciente de errores u olvidos en el registro o la difusión de datos privados.

d) Protección universal de todos los datos de todos los pacientes en todos los centros.

Puesto que la historia clínica es una colección de datos personales que se almacenan en un fichero, además de regularse por la Ley de Autonomía del Paciente y la Ley Foral 17/2010, debemos atenernos a lo dispuesto en la Ley de Protección de Da$\operatorname{tos}^{24}$ en lo que a recogida y tratamiento de la información se refiere.

\section{NATURALEZA JURÍDICA, NECESIDAD Y CONTENIDO DE LA HISTORIA CLÍNICA}

La legislación actual no aclara la naturaleza jurídica de la historia clínica si bien la doctrina jurisprudencial la ha venido considerando tradicionalmente como un documento privado.

La historia clínica puede ser usada como prueba en procesos judiciales y en muchas ocasiones determina las decisiones que se toman, estimando su valor probatorio para demostrar la verdad en el proceso; no sólo lo que concierne a la relación o actos sobre el paciente, sino al cumplimiento de preceptos como la solicitud del consentimiento informado, deber de asistencia, etc. ${ }^{25}$. Una de las causas por las que se le presupone veracidad es que se redacta en el momento de la atención y no a posteriori cuando surge la demanda.

La necesidad de crear y utilizar una historia clínica es obvia, especialmente en nuestros días, en los que múltiples profesionales de diferentes estamentos y especialidades deben implicarse en la atención al paciente, amén de realizarse gran cantidad de pruebas complementarias. De todo ello debe quedar constancia por el deber ético de beneficencia hacia el paciente, pues la falta de información dificultaría o impediría dispensar con garantías los cuidados adecuados al paciente en un momento concreto. También la Ley de Autonomía del Paciente indica el derecho del paciente 
a que se recojan todos los datos relativos a su proceso (art. 15.1) y el deber de los profesionales de colaborar en su creación y mantenimiento (art. 17.3).

En la tabla 1 se pueden ver los contenidos mínimos que debe tener la historia clínica según la Ley Foral 17/2010. Cabe destacar en lo que respecta a enfermería que se incluye necesariamente la documentación referente a sus tareas y a los procesos enfermeros, ampliando el concepto tradicional puramente "médico" de la historia clínica.

Podemos saber los documentos que se deben incluir, pero no se especifican directrices formales en cuanto a su cumplimentación. Para respetar al máximo la intimidad y dignidad de los pacientes, éticamente el profesional debería imponerse una serie de normas en la redacción de la historia clínica para conseguir las siguientes características:
- Completa, con datos suficientes y sintéticos sobre el proceso, pruebas complementarias, etc.

- Ordenada cronológicamente en las anotaciones y documentos adjuntos.

- Inteligible, tanto ortográfica como gramaticalmente.

- Identificativa del profesional que escribe, con sus datos personales y firma en caso de ser manuscrita ${ }^{26}$.

Cabe destacar también que la Ley de Protección de Datos parte del principio básico de la exigibilidad del consentimiento del afectado para el tratamiento de sus datos de carácter personal (art. 6.1), si bien no sería preciso el consentimiento de los pacientes que acuden al centro sanitario, por lo que el profesional cumpliría lo establecido limitándose a informar al interesado de la existencia del tratamiento de sus datos personales y de los posibles destinatarios de los mismos (art. 11).

Tabla 1. Contenido mínimo de la historia clínica según la Ley Foral 17/2010

\begin{tabular}{|c|c|}
\hline \multicolumn{2}{|c|}{ A) Datos de identificación del enfermo y de la asistencia } \\
\hline $\begin{array}{l}\text { - Nombre y apellidos del enfermo. } \\
\text { - Fecha de nacimiento. } \\
\text { - Sexo. } \\
\text { - Código de identificación personal contenido en la } \\
\text { tarjeta sanitaria individual. } \\
\text { - Domicilio habitual y teléfono. } \\
\text { - Fecha de asistencia y de ingreso. }\end{array}$ & $\begin{array}{l}\text { - Indicación de la procedencia, en caso de derivación } \\
\text { desde otro centro asistencial. } \\
\text { - Servicio o unidad en que se presta la asistencia.* } \\
\text { - Número de habitación y de cama, en caso de ingreso. } \\
\text { - Médico responsable del enfermo. }\end{array}$ \\
\hline \multicolumn{2}{|c|}{ B) Datos clínico-asistenciales } \\
\hline $\begin{array}{l}\text { - Antecedentes familiares y personales fisiológicos y } \\
\text { patológicos. } \\
\text { - Descripción de la enfermedad o del problema de } \\
\text { salud actual y motivos sucesivos de consulta. } \\
\text { - Procedimientos clínicos empleados y sus resultados, } \\
\text { con los dictámenes correspondientes emitidos en } \\
\text { caso de procedimientos o exámenes especializados y } \\
\text { también las hojas de interconsulta. } \\
\text { - Hojas de curso clínico, en caso de ingreso. } \\
\text { - Hojas de tratamiento médico. } \\
\text { - Hoja de consentimiento informado.* } \\
\text { - Hoja de información facilitada al paciente en relación } \\
\text { con el diagnóstico y el plan terapéutico prescrito.* } \\
\text { - Informes de epicrisis o de alta, en su caso. }\end{array}$ & $\begin{array}{l}\text { - Documento de alta voluntaria, en su caso. } \\
\text { - Informe de necropsia, si existe. } \\
\text { - En caso de intervención quirúrgica, debe incluirse la } \\
\text { hoja operatoria y el informe de anestesia, y en caso de } \\
\text { parto, los datos de registro. } \\
\text { - El informe de urgencia. } \\
\text { - La autorización de ingreso. } \\
\text { - El informe de anatomía patológica. } \\
\text { - En su caso, el documento de voluntades anticipadas, } \\
\text { así como posible condición de donante de órganos. } \\
\text { - La evolución y planificación de los cuidados de } \\
\text { enfermería. } \\
\text { - La aplicación terapéutica de enfermería. } \\
\text { - El gráfico de constantes. } \\
\text { - El informe clínico de alta. }\end{array}$ \\
\hline
\end{tabular}

C) Datos sociales

- Informe social.*

* Si procede.

An. Sist. Sanit. Navar. 2011, Vol. 34, No 1, enero-abril 


\section{PROPIEDAD, ACCESO Y USOS DE LA HISTORIA CLÍNICA}

La ley no especifica de quien es propiedad la historia clínica y este tema ha generado debate con tres posiciones fundamentales $^{27-29}$.

- Propiedad del paciente. La información que consta en la historia clínica se refiere al paciente y ha sido cedida por él mismo en primera persona o con su consentimiento a través de pruebas diagnósticas.

- Propiedad del profesional. La historia redactada por el profesional es fruto directo de su trabajo intelectual y esta autoría le otorga derecho de propiedad.

- Propiedad del centro sanitario (excepto en profesionales que trabajan por cuenta propia). Se basa en que si bien el profesional es el autor, el empleador le paga por su trabajo en el que se incluye la cumplimentación de la historia clínica, que se considera un producto de su actividad laboral.

En cualquier caso, más que la propiedad, el derecho subyacente en este debate es el del acceso a los datos contenidos en la historia clínica, por lo que cabría mejor hablar de titularidad del derecho de acceso, que sería compartida en los términos en que determina la legislación ${ }^{26}$.

La Sociedad Española de Medicina Familiar y Comunitaria propone que la información contenida en la historia clínica se estructure en tres niveles:

1. Datos básicos a los que cualquier profesional pueda tener acceso en la atención del paciente.

2. Datos privados a los que sólo se podría acceder con el consentimiento expreso del paciente.

3. Datos reservados a los que el paciente no tendría acceso. Incluiría anotaciones subjetivas del profesional o datos que conciernan a terceras personas $^{30}$.

La Ley de Autonomía del Paciente establece que el acceso a la historia clínica es libre por parte de los profesionales sanitarios, pero exclusivamente en cuanto a los datos pertinentes para garantizar una asistencia adecuada al paciente y en tanto en cuanto los datos de la historia constituyan un "instrumento fundamental para su adecuada asistencia" en cada caso concreto. No es posible especificar de manera genérica qué es pertinente o no pues la casuística es amplísima y habría que analizar cada caso particularmente para determinar la relación con el mismo, si bien debe interpretarse el acceso en los términos que resulten más beneficiosos para lograr la mejor asistencia sanitaria del paciente ${ }^{31}$. Por ejemplo, imaginemos un paciente que acude a su centro de salud a quitarse unos puntos por un corte en la muñeca. Sería muy difícil de entender para él que su enfermera consultara en su historia clínica sus antecedentes psiquiátricos. Puede que haya relación (o no), pero en ningún caso tiene utilidad para la asistencia en sí.

En el caso de los pacientes (o un representante debidamente acreditado), se reconoce su derecho a acceder a los datos contenidos en su historia clínica y a obtener copia de ellos, excepción hecha de los que hagan referencia a terceras personas por suponer una violación de la intimidad de éstas $^{32}$. Además los profesionales participantes en su elaboración pueden oponer al derecho de acceso la reserva de sus anotaciones subjetivas. También tienen derecho "a conocer en todo caso quién ha accedido a sus datos sanitarios, el motivo del acceso y el uso que se ha hecho de ellos, salvo en caso del uso codificado de los mismos" (Ley Foral 17/2010 art. 31.1). En caso de fallecimiento, el acceso a la historia clínica solo se permitirá "a las personas vinculadas a él, por razones familiares o de hecho, salvo que el fallecido lo hubiese prohibido expresamente". En caso de existir un riesgo para la salud de un tercero, éste puede tener acceso a la historia clínica, pero se limitará a los datos pertinentes. No se facilitará información que afecte a la intimidad del fallecido ni a las anotaciones subjetivas de los profesionales, ni que perjudique a terceros. (Ley de Autonomía del Paciente, art 18.3 y 18.4). 
La Ley de Autonomía del paciente no aclara qué se entiende por anotaciones subjetivas. Este es uno de los puntos más controvertidos cuando se habla de qué puede y qué no puede ver el paciente por su indefinición que quedaba pendiente de definición ${ }^{30}$. De ello se ha encargado la Ley Foral 17/2010 que lo expresa literalmente así en el artículo 64.4:

\footnotetext{
"A los efectos de lo dispuesto en la presente Ley Foral, se entenderán por anotaciones subjetivas las impresiones o valoraciones personales de los profesionales sanitarios no sustentadas directamente en datos objetivos o pruebas complementarias y que, en su criterio, resulten de interés para la atención sanitaria del paciente. Se considerarán anotaciones subjetivas únicamente aquellas que puedan encuadrarse en algunos de los siguientes apartados:

- Valoraciones sobre hipótesis diagnósticas no demostradas

- Sospechas acerca de incumplimientos terapéuticos

- Sospechas de tratamientos no declarados

- Sospechas de hábitos no reconocidos

- Sospechas de haber sido víctima de malos tratos

- Comportamientos insólitos
}

Los profesionales sanitarios deberán abstenerse de incluir expresiones, comentarios o datos que no tengan relación con la asistencia sanitaria del paciente o que carezcan de valor sanitario".

El uso inicial y fundamental de la historia clínica es el asistencial pero se consideran también una serie de usos con fines diferentes a este: fines judiciales, epidemiológicos, de salud pública, de investigación y docentes. En estos casos debe disociarse la información personal del paciente de la asistencial para garantizar su anonimato (excepto orden judicial en contra o consentimiento del paciente). Además se regirán por lo dispuesto en la Ley de Protección de Datos, la Ley General de Sanidad y demás normas de aplicación en cada caso (Ley de Autonomía del Paciente, art. 16).

También se contemplan fines de administración, de inspección, de evaluación, de acreditación y de planificación.
Como norma general debe garantizarse que el profesional que atiende al paciente tenga garantizado siempre el acceso a la historia clínica del mismo.

Para conseguir algunos de esos fines, el acceso a la historia clínica se realizará por parte de personal no sanitario. En cualquier caso, toda persona que acceda a esta información queda obligada a guardar secreto profesional por todas las razones éticas expuestas en la primera parte y porque así lo indica la ley.

En el caso de las actividades docentes en las que se usa gran cantidad sobre todo de imágenes, los discentes también están obligados al secreto si apareciera información sobre el paciente. Afortunadamente esto es cada vez menos habitual ${ }^{20,23}$.

No es algo conocido por todos los profesionales sanitarios que en caso de no guardar el debido secreto, se prevén una serie de sanciones ${ }^{17}$. Así la Ley de Protección de Datos en su artículo 44.4.B considera infracción muy grave la vulneración del deber de guardar secreto sobre datos referentes a la salud, sancionada con multa de entre $300.000,00$ y 600.000,00 euros.

El artículo 199.2 del código penal dice "El profesional que, con incumplimiento de su obligación de sigilo o reserva, divulgue los secretos de otra persona, será castigado con la pena de prisión de uno a cuatro años, multa de doce a veinticuatro meses e inhabilitación especial para dicha profesión por tiempo de dos a seis años". Y en ese sentido se ha pronunciado el Tribunal Supremo ${ }^{33,34}$.

\section{CUSTODIA, CONSERVACIÓN Y RECTIFICACIÓN DE LA HISTORIA CLÍNICA}

La custodia de la historia clínica en centros sanitarios es responsabilidad del centro y personal en caso de profesionales que trabajan por cuenta propia.

Se entiende por custodia la conservación de los datos de la historia clínica (independientemente del soporte en que se haga) y por otro la garantía de acceso a los 
profesionales del centro en cualquier momento por necesidad asistencial. Esta tarea correrá a cargo de las unidades de admisión y documentación clínica que deberán integrar en un solo archivo las historias clínicas.

En la actualidad con la informatización de los archivos, corresponde también al centro el uso de medidas de seguridad que garanticen la confidencialidad y autenticidad de los datos, a la vez que registren los accesos a los mismos y la identidad de los creadores en cada momento. Cuando el volumen de datos así lo aconseje estas tareas podrán ser externalizadas a empresas privadas, si bien la titularidad de la responsabilidad sobre los datos y su tratamiento seguirá siendo del centro. Para más información sobre las medidas de seguridad que deben tener los ficheros, se puede consultar el Real Decreto 1720/2007, de 21 de diciembre, por el que se aprueba el Reglamento de desarrollo de la Ley Orgánica 15/1999, de 13 de diciembre, de protección de datos de carácter personal.

Los datos referentes a un proceso asistencial deben conservarse al menos durante los 5 años siguientes al alta del mismo (Ley de Autonomía del Paciente art. 17.1). Si bien este mandato es claro, en el punto siguiente matiza que también debe conservarse a efectos judiciales de conformidad con la legislación vigente y por razones epidemiológicas, de investigación o de organización y planificación. No queda pues claro cuándo puede destruirse la historia clínica ni qué partes de la misma.

La cancelación de datos de la historia clínica por solicitud del afectado no se realiza de forma automática. La Ley de Autonomía del Paciente impone el tratamiento de datos en la atención de procesos de salud y la Ley de Protección de Datos permite recogerlos incluso sin el consentimiento del interesado. Además no es posible la cancelación de los datos necesarios para el adecuado tratamiento del paciente o de aquéllos que el responsable del archivo está legalmente obligado a mantener, recuérdese, por el plazo que resulte pertinente, nunca inferior a cinco años ${ }^{35,37}$. No obstante, en base a los principios de pertinencia y proporcionalidad, podrían cancelarse datos que no fueran pertinentes para el tratamiento en el proceso asistencial ${ }^{38}$. Estos temas se prestan a diversas interpretaciones y no existe entre los autores unanimidad al respecto.

La Ley de Protección de Datos ampara el derecho a "ocultar" datos especialmente sensibles o aquellos que se consideren irrelevantes tras cierto periodo de tiempo, de modo que sin ser borrados (para no faltar al deber de custodia) no sean accesibles. Con la informatización de la historia clínica esta práctica es posible, pero no hay una definición clara de cuáles pueden ser esos datos ni a quien corresponde hacerlo ${ }^{16,39}$.

Se contempla la solicitud de rectificación, que deberá indicar el dato que es erróneo y la corrección que debe realizarse y deberá ir acompañada de la documentación justificativa de la rectificación solicitada.

\section{CONSIDERACIONES FINALES}

Como profesionales de la salud es necesario tener presente la necesidad de preservar la confidencialidad de los datos de nuestros pacientes. En nuestro ámbito cultural existe cierto relajo en la observancia de las normas del secreto profesional ya sea por descuido, divulgación o por acceso indebido a la información. Muchas veces no se le da la importancia que merece y no se tiene conciencia de la magnitud de las consecuencias que puede tener para ambas partes. Todo aquel que llegue a conocer datos confidenciales está obligado al secreto y por muchas contraseñas o trabas que se pongan, la última barrera es la propia discreción.

Se impone la necesidad de un cambio en la cultura del manejo de datos, así como de hacer mayor énfasis sobre este tema en la formación de los futuros profesionales.

La historia clínica es un elemento imprescindible e ineludible para garantizar la calidad de la atención sanitaria. Es el documento que refleja el quehacer del profesional en pro de la salud del paciente. En ella 
queda la impronta de quien la realiza y por ello es necesario tener en cuenta principios éticos y formales a la hora de su redacción. Recoger los datos justos y necesarios para la atención al paciente, desechando lo superfluo o improcedente debe ser un aspecto más de la excelencia clínica.

Conocer las múltiples implicaciones éticas y legales que atañen a la historia clínica ayuda al profesional en su trabajo y en la relación con los pacientes.

\section{BIBLIOGRAFÍA}

1. Consejo Internacional de Enfermeras. Código Deontológico del CIE para la profesión de Enfermería. Ginebra 2006.

2. Organización de Naciones Unidas. Declaración Universal de Derechos Humanos. [3/12/2010] http://www.un.org/es/documents/udhr/index.shtml.

3. Constitución Española. BOE 29-12-1978, 331: 29313-29424.

4. Instrumento de Ratificación del Convenio para la protección de los derechos humanos y la dignidad del ser humano con respecto a las aplicaciones de la Biología y la Medicina (Derechos humanos y Biomedicina), Oviedo 4-4-97. BOE 20-10-99; 251: 36825-36830.

5. Ley $14 / 1986$, de 25 de abril, General de Sanidad. BOE 29-4/-86; nº 102: 15207-15224.

6. Ley Orgánica 15/1999, de 13 de diciembre, de Protección de Datos de Carácter Personal. BOE 14-12-99; 298: 43088-43099.

7. Real Decreto $1720 / 2007$, de 21 de diciembre, por el que se aprueba el Reglamento de desarrollo de la Ley Orgánica 15/1999, de 13 de diciembre, de protección de datos de carácter personal. BOE 19-1-08; 17: 41034136.

8. Ley 41/2002, de 14 de noviembre, básica reguladora de la autonomía del paciente y de derechos y obligaciones en materia de información y documentación clínica. BOE 15-1102; 274: 40126-40132.

9. Ley Foral 17/2010, de 8 de noviembre, de derechos y deberes de las personas en materia de salud en la Comunidad Foral de Navarra. BON 15-11-10; 139: 15023-15036.

10. Ley Foral 11/2002, de 6 de mayo, sobre los derechos del paciente a las voluntades anticipadas, a la información y a la documentación clínica. BON 13-05-2002; 58: 4224-4227.
11. Consejo General de Colegios Médicos. Código de ética y deontología médica 1999. [4/12/2010]; http://nueva.cgcom.es/deontologia

12. Consejo General de Enfermería. Código deontológico de la enfermería española. 1998 [4/12/2010];http://www.enfermerianavarra.com/

13. Real Academia Española. Diccionario de la lengua española. $22^{\mathrm{a}}$ Ed. www.rae.es. Consultado el 28/11/2010.

14. Fernández Lamelas MA, Álvarez Rodríguez T, Ramiro Fernández JM, Martínez de Santiago S. El respeto a la intimidad. El secreto profesional en Enfermería. Cuad Bioet 2008; 19: 59-66.

15. CRiado del Río Mt. Aspectos médico-legales de la historia clínica. Med Clin 1999; 112: 24-28.

16. Iraburu M. Confidencialidad e intimidad. An Sist Sanit Navar 2006; 29 Suppl 3: 49-59.

17. Iraburu M, Chamorro J, de Pedro MT. Conocimientos, comportamientos y opiniones de los profesionales sanitarios de un hospital en relación a la confidencialidad. An Sist Sanit Navar 2006; 29: 357-366.

18. Hasman A, Hansen NR, Lassen A, Rabol R, Holm S. What do people talk about in Danish hospital elevators? Ugeskr Laeger 1997; 159: 6819-6821.

19. Vigod SN, Bell CM, Bohnen JM. Privacy of patients information in hospital lifts: observational study. BMJ 2003; 327: 1024-1025.

20. Heitzmann T. El secreto médico: actitudes y toma de decisiones en la práctica clínica (tesis doctoral). Universidad Complutense Madrid; 2003.

21. Perez-Carceles MD, Lorenzo MD, Luna A, Osuna E. Elderly patients also have rights. J Med Ethics 2007; 33: 712-716.

22. Perez-Carceles mD, Pereniguez Je, Osuna E, Perez-Flores D, Luna A. Primary care confidentiality for Spanish adolescents: fact or fiction? J Med Ethics 2006; 32: 329-340.

23. IRABURU M. Estudio multicéntrico de investigación sobre la confidencialidad. Med Clin 2007; 128: 575-578.

24. Ley Orgánica 15/1999, de 13 de diciembre, de Protección de Datos de Carácter Personal. BOE 14-12-99; 298: 43088-43099.

25. Sarrato Martínez L. La historia clínica y su acceso con fines judiciales. Diario la Ley. 4-108; XXIX 6854.

26. MÉJica García J, Bobes García J. La ley del paciente para profesionales sanitarios. $2 \mathrm{Ed}$. Méjica F, Editor. Gijón: Ojo X Hoja; 2009. 
27. De Lorenzo L, Montero R. Responsabilidad legal del profesional sanitario. Edicomplet, Madrid: Asociación Española de Derecho Sanitario; 2000.

28. Martínez Hernández J. Historia Clínica. Cuad Bioét 2006; 1․ 57-68.

29. Porteo Lazcano G. Historia clínica: Problemática sobre la propiedad. Rev Lationam Der Med Medic Leg 2002; 6-7 (2-1): 81-88.

30. semFYC. Grupo de Trabajo de Bioética de la semFYC. Declaración de la Sociedad Española de Medicina de Familia y Comunitaria. Informatización y confidencialidad en la historia clínica. Aten Primaria 2004; 34: 140-142.

31. Gabinete jurídico de la Agencia Española de Protección de Datos. Informe 656/2008 Acceso a historia clínica por personal de enfermería 2009: Available from: www.agpd.es.

32. Agencia de Protección de Datos de la Comunidad de Madrid. Resolución. Solicitada la historia clínica de una persona fallecida, por parte de sus familiares, en la documentación entregada figuran los datos personales relativos a otros pacientes. 25-02-2010; http:// www.madrid.org.

33 Ley Orgánica 10/1995, de 23 de noviembre, del Código Penal. BOE 24-11-1995; 281: 3398734058 .
34. Tribunal Supremo, Sala de lo Penal. Sentencia $n^{\circ} 574 / 2001$. Condena a un médico cuya indiscreción provocó una violación del secreto profesional 2001; Vista: 27-3-01.

35. Gabinete jurídico de la Agencia Española de Protección de Datos. Informe 189/2003 Cancelación de datos contenidos en historias clínicas.2003; www.agpd.es.

36. Agencia de Protección de Datos de la Comunidad de Madrid. Resolución "La solicitud de rectificación de datos debe justificarse con la acreditación del carácter erróneo de los mismos" 25-9-09; http://www.madrid.org/.

37. Agencia de Protección de Datos de la Comunidad de Madrid. Resolución "Estimación del derecho de acceso y desestimación de derecho de cancelación"24/09/2009; http://www. madrid.org.

38. Gabinete jurídico de la Agencia Española de Protección de Datos. Informe 0173/2008 Contenido de la historia clínica (proporcionalidad) 2008; www.agpd.es.

39. Delgado Marroquín MT. Quiero que borre de mi historia el antecedente de aborto. Aten Primaria 2005; 36: 339-343. 